\title{
TEACHING INTELLIGENT CONTROL: THE INVERTED PENDULUM EXPERIMENT REVISITED
}

Alexandre Alves Santiago ${ }^{1}$, Glauber Bolzan de Assis Lima²

DOI: 10.37702/REE2236-0158.v39p89-98.2020

\begin{abstract}
This work shows how to use inverted pendulum hardware in the teaching of intelligent control techniques. The concepts of a fuzzy logic based controller are applied on an inverted pendulum. The design process is explained in detail, offering a practical guide for the reproduction of both the experiment and simulation. Last but not least, the results are commented and the performance achieved with this controller is compared to that of the original one, which was delivered with the hardware. This work also suggests future developments for new undergraduate teams.
\end{abstract}

Keywords: Engineering Teaching; Intelligent Control; Inverted Pendulum.

\section{INTRODUCTION}

This undergraduate project was carried out by two students of the CEFET-RJ Institute of Technology as a partial requirement for the Bachelor Degree in Control and Automation Engineering (LIMA; BELARMINDO, 2011).

The motivation this teaching experience consists of using the inverted pendulum hardware to demonstrate the limitations of mathematical modeling and the application of robust intelligent control techniques to a complex system.

The inverted pendulum is an inherently unstable mechanical system, constituting a benchmark for the study of many control techniques. The dynamics of an inverted pendulum is applicable to more complex systems like heavy rocket vertical stabilization at the beginning of the launch phase, skyscrapers oscillation control, walking robots, biomechanics and many others (BLOCK; ÄSTRÖM; SPONG, 2007).

The experimental setup consists of a High Fidelity Linear Cart, HFLC, developed by
Quanser (QUANSER, 2008). This device consists of an inverted pendulum assembled on a cart. The HFLC has a solid aluminum cart driven by a $400 \mathrm{~W}$ brushless DC motor, which is connected by pinion and rack to a $0.8 \mathrm{~m}$ rail, therefore eliminating slippage and belt stretching usually found in belt transmission systems. The cart's position with respect to the rail is measured by a high precision encoder.

Figure 1 - Inverted pendulum HFLC Quanser

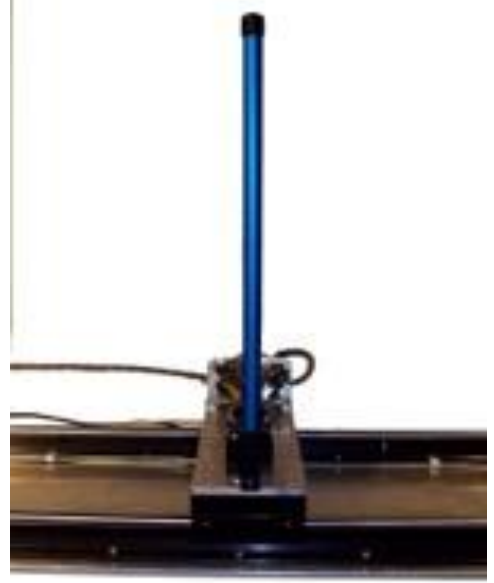

Source: Quanser, 2008. 
As a result, this system is able to deliver high accuracy, speed, acceleration, and excellent repeatability. The cart, pendulum, and part of the rail can be seen in Figure 1.

When the Inverted Pendulum HFLC was first put into operation, some problems were detected at the swing-up phase. At each time the system was turned on, the swing-up controller caused the car to hit the limit switches at the rail tips, therefore interrupting the operation. In this way, system behavior turns out to be unpredictable.

The reason for this abnormal behavior was found to be in the swing-up controller originally adopted at the HFLC design, which was based on an energy control approach proposed by Furuta (BLOCK; ÄSTRÖM; SPONG, 2007). This technique was based on hardware called rotational inverted pendulum as shown in Figure 2, which differs considerably from the linear cart based inverted pendulum as shown in Figure 1.

Figure 2 - Rotational inverted pendulum

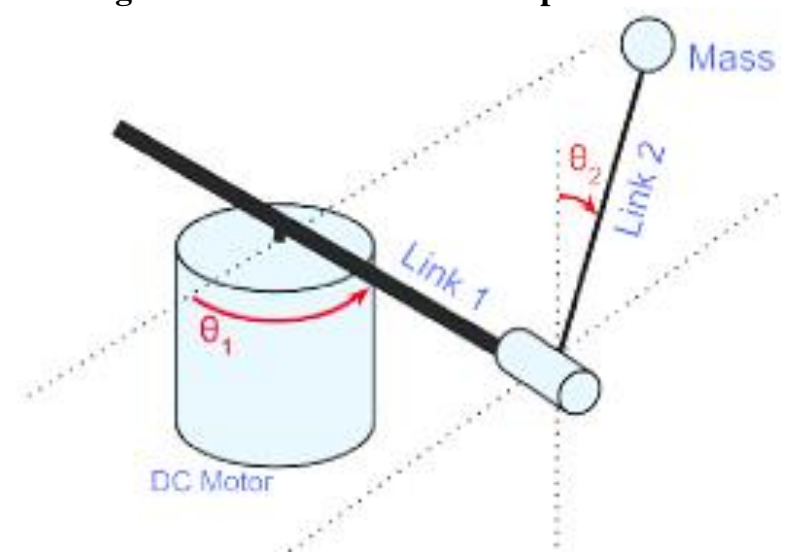

Source: elaborated by the authors.

In the rotational pendulum, as shown in Figure 2, the horizontal bar can turn indefinitely around the vertical axis reaching any value of $\theta_{1}$.

The Furuta's swing-up control applied to HFLC design takes into account only two inputs, $\theta$ and $\dot{\theta}$, and one output, in this case, the current $u$ applied to the car motor. The cart position along the rail was not taken into account, which leads to collision against the rail limits.

In the present work, the inverted pendulum has its pivot fixed on a cart, which moves along a straight horizontal rail, being the longitudinal position control of the cart over a limited rack length of paramount importance. In this way, the swing-up algorithm adopted by Furuta doesn't apply to the Quanser design, whose cart motion is limited by a rail with $0.8 \mathrm{~m}$ length.

\section{THE INVERTED PENDULUM DYNAMICS}

\section{The pendulum parameters}

According to the User Manual (QUANSER, 2008) and to the Figure 3, the following parameters will be considered in the dynamic modelling of the inverted pendulum:

$x$, position of the cart;

$\theta$, angle of the pendulum;

$M_{p}=0.23 \mathrm{~kg}$, mass of the pendulum;

$M_{c}=3.22 \mathrm{~kg}$, mass of the cart;

$l_{p}=0.3302 \mathrm{~m}$, distance between the pendulum's pivot and its center of mass $G$;

$L_{p}=0.6413 \mathrm{~m}$, pendulum length;

$B_{p}=2.40\left(10^{-3}\right) \mathrm{Nms} / \mathrm{rad}$, pendulum's pivot damping coefficient.

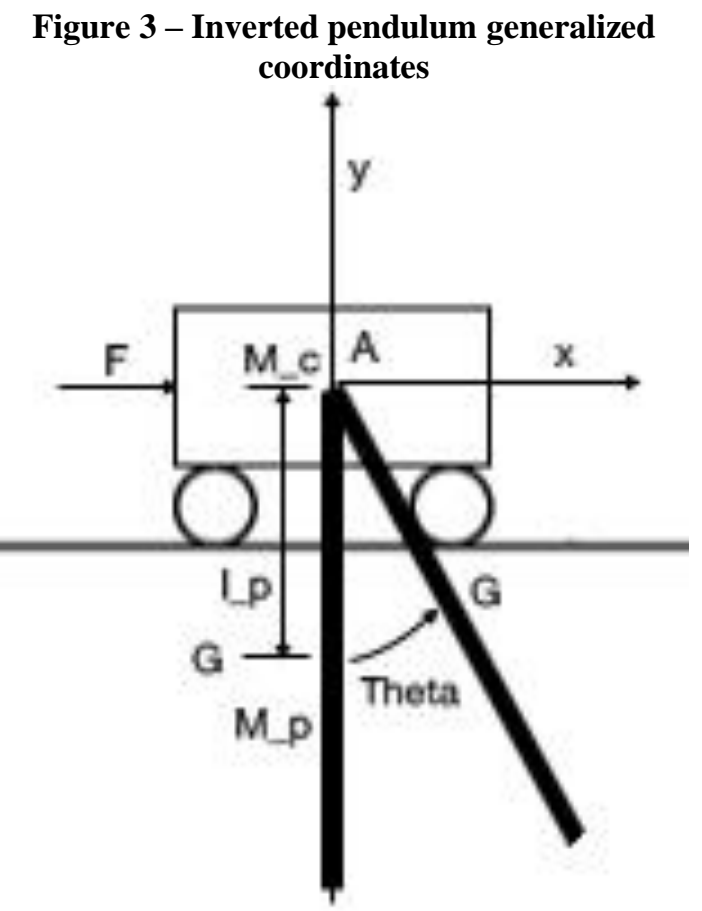

Source: elaborated by the authors. 


\section{Dynamic model equations}

The derivation of the inverted pendulum dynamics is carried out according to the Lagrangian Theorem (MERIAM, 1966) shown in equation 1 ,

$\frac{d}{d t}\left(\frac{\partial L}{\partial \dot{q}_{l}}\right)-\frac{\partial L}{\partial q_{i}}=Q_{i}$

where:

$L=T-V$ is the difference between kinetic $\mathrm{T}$ and the potential energy $\mathrm{V}$; and

$q_{i}$ is the $i^{t h}$ generalized coordinate;

$Q_{i}$ is the $i^{\text {th }}$ torque or force input related to a given coordinate.

According to Figure 3, the generalized coordinates are $q_{1}=x$ and $q_{2}=\theta$. The generalized forces are $Q_{1}=F$ applied to the cart, and the torque $Q_{2}=-B_{p} \dot{\theta}$ acting at the pendulum pivot where $B_{p}$ is the damping coefficient.

The speed of the pendulum center of gravity is given by Equation (4).

$\overrightarrow{V G}=\overrightarrow{V A}+\dot{\theta} \hat{k} \times l_{P}(\sin \theta \hat{\imath}-\cos \theta \hat{\jmath})$

$\overrightarrow{V A}=\dot{x} \hat{\imath}$

$\overrightarrow{V G}=\left[\dot{x} \dot{\theta} l_{p} \cos \theta\right] \hat{\imath}+\dot{\theta} l_{p} \sin \theta \hat{\jmath}$

where $l_{p}$ is the distance between the pivot and the pendulum's center of mass.

The potential energy $V$ of the system is described as follows:

$$
V=-M_{p} g l_{p} \cos \theta
$$

The kinetic energy of the system is described by:

$$
T=\frac{1}{2} M_{p} V_{G}^{2}+\frac{1}{2} J_{p} \theta^{2}+\frac{1}{2} M_{c} \dot{x}^{2}
$$

where: $M_{p}$ is the pendulum's mass; $J_{p}$ is the pendulum's barycentric moment of inertia; and $M_{C}$ is the cart's mass.

The substitution of equation (4) in (6) leads to (7)

$$
\begin{aligned}
& T=\frac{1}{2}\left[M_{p}+M_{c}\right] \dot{x}^{2}+M_{p} \dot{x} \dot{\theta} l_{p} \cos \theta+ \\
& \frac{1}{2}\left[M_{p} l_{p}^{2}+J_{p}\right] \dot{\theta}^{2}
\end{aligned}
$$

The Lagrangian $L$ of the system is denoted by,

$$
\begin{aligned}
L & =T-V \\
L & =\frac{1}{2}\left[M_{p}+M_{c}\right] \dot{x}^{2}+M_{p} \dot{x} \dot{\theta} l_{p} \cos \theta+ \\
\frac{1}{2}\left[M_{p} l_{p}^{2}\right. & \left.+J_{p}\right] \dot{\theta}^{2}+M_{p} g l_{p} \cos \theta
\end{aligned}
$$

Deriving the terms of Equation (1) with respect to the first generalized variable $x$, as follows,

$$
\begin{gathered}
\frac{\partial L}{\partial x}=0 \\
\frac{\partial L}{\partial \dot{x}}=\left[M_{p}+M_{c}\right] \dot{x}+M_{p} \dot{\theta} l_{p} \cos \theta \\
\frac{d}{d t}\left(\frac{\partial L}{\partial \dot{x}}\right)=\left[M_{p}+M_{c}\right] \ddot{x}+M_{p} L_{p} \ddot{\theta} \cos \theta- \\
M_{p} l_{p} \sin \theta \dot{\theta}^{2}
\end{gathered}
$$
follows,

Substituting (10), (11), and (12) in (1)

$$
\begin{gathered}
\frac{d}{d t}\left(\frac{\partial L}{\partial \dot{x}}\right)-\frac{\partial L}{\partial x}=F \\
{\left[M_{p}+M_{c}\right] \ddot{x}+M_{p} l_{p} \ddot{\theta} \cos \theta-} \\
M_{p} l_{p} \sin \theta \ddot{\theta}^{2}=F
\end{gathered}
$$

With respect to the second generalized variable $\theta$ follows,

$$
\begin{array}{r}
\frac{\partial L}{\partial \theta}=-M_{p} \dot{x} \dot{\theta} l_{p} \sin \theta-M_{p} g l_{p} \sin \theta \\
=-M_{p} l_{p} \sin \theta[\dot{x} \dot{\theta}+g] \\
\frac{\partial L}{\partial \dot{\theta}}=M_{p} \dot{x} l_{p} \cos \theta+\left[M_{p} l_{p}^{2}+J_{p}\right] \ddot{\theta} \\
\frac{d}{d t}\left(\frac{\partial L}{\partial \dot{\theta}}\right)=M_{p} \ddot{x} l_{p} \cos \theta- \\
M_{p} l_{p} \dot{x} \dot{\theta} \sin \theta+\left[M_{p} l_{p}^{2}+J_{p}\right] \ddot{\theta}
\end{array}
$$
follows,

Substituting (16), (17), and (18) in (1)

$$
\begin{aligned}
& \frac{d}{d t}\left(\frac{\partial L}{\partial \dot{\theta}}\right)-\frac{\partial L}{\partial \theta}=-B_{p} \dot{\theta} \\
& M_{p} \ddot{x} l_{p} \cos \theta-M_{p} l_{p} \dot{x} \dot{\theta} \sin \theta+ \\
& {\left[M_{p} l_{p}^{2}+J_{p}\right] \ddot{\theta}+M_{p} l_{p} \dot{x} \sin \theta+} \\
& M_{p} g l_{p} \sin \theta=-B_{p} \dot{\theta}
\end{aligned}
$$


so that the following differential equations describe the inverted pendulum dynamics,

$$
\begin{aligned}
& {\left[M_{p}+M_{c}\right] \ddot{x}+M_{p} l_{p} \cos \theta \ddot{\theta}=} \\
& M_{p} l_{p} \sin \theta \dot{\theta}^{2}+F \\
& M_{p} l_{p} \cos \theta \ddot{x}+\left[M_{p} l_{p}^{2}+J_{p}\right] \ddot{\theta}= \\
& -M_{p} g l_{p} \sin \theta-B_{p} \dot{\theta}
\end{aligned}
$$

The equations (21) and (22) are then expressed in state space form,

$$
\begin{aligned}
{\left[\begin{array}{l}
\ddot{x} \\
\ddot{\theta}
\end{array}\right]=\left[\begin{array}{cc}
\left(M_{p}+M_{c}\right) & \left(M_{p} l_{p} \cos \theta\right) \\
\left(M_{p} l_{p} \cos \theta\right) & \left(M_{p} l_{p}^{2}+J_{p}\right)
\end{array}\right]^{-1} } \\
{\left[\begin{array}{c}
\left(M_{p} l_{p} \sin \theta \dot{\theta}^{2}+F\right) \\
\left(-M_{p} l_{p} g \sin \theta-B_{p} \dot{\theta}\right)
\end{array}\right] }
\end{aligned}
$$

\section{THE FUZZY CONTROLLER}

\section{Control Architecture}

The complete control of an inverted pendulum usually requires three steps:

- the swing-up control to invert the pendulum position upwards;

- the position control to keep the pendulum upward and inverted; and

- the transition control to switch between swing-up and controlled position.

The swing-up phase consists of moving the pendulum bar upwards from a rest condition, without applying torque directly at its pivot. Moving the cart to right and left causes the bar to oscillate with increasing amplitude, generating the swing motion. The cart, therefore, excites the pendulum at its natural frequency, consequently raising its mechanical energy. The diagram at Figure 4 illustrates this condition.
Figure 4 - Inverted pendulum system motion

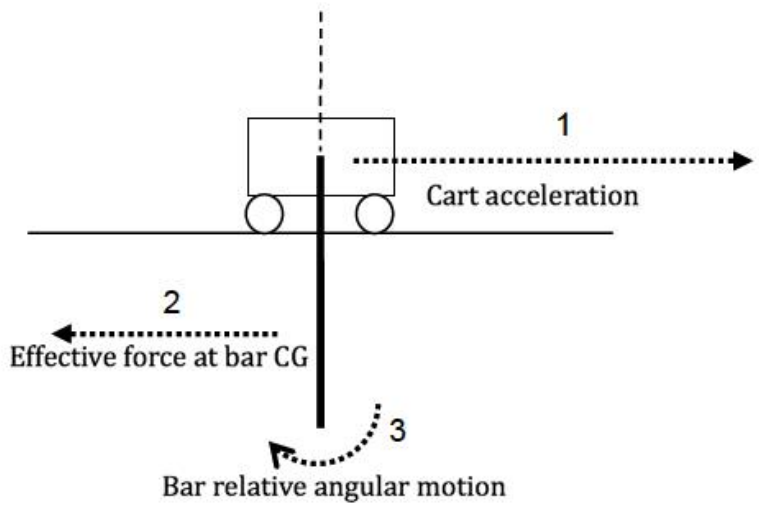

Source: elaborated by the authors.

\section{The heuristic fuzzy swing-up design}

The first approach to design a fuzzy controller consists in replicating the behavior of a human operator by creating a set of rules that reproduces his or her control actions, and this procedure was followed in this work.

The pendulum swing-up was performed many times by hand until this task was successfully accomplished, thus establishing the following steps:

- The system begins at rest with the cart at rail's center and the pendulum pointing downwards at zero angle, as shown in Figure 5.

- With this first move to the left, in this case, the pendulum begins its swing.

- When the bar comes again to zero angle, its rotation is clockwise and the cart then moves to the right of the rail, avoiding impacting at the rail limit. In this way, the bar swing amplitude increases. This sequence is shown in Figure 6.

- Once the car reaches the right end position, it stops until the pendulum reaches zero angle and counterclockwise rotation. Once this condition is reached, the car moves once more to the left end, thus increasing the swing amplitude and mechanical energy of the bar, as shown in Figure 7. 
Figure 5 - First cart motion

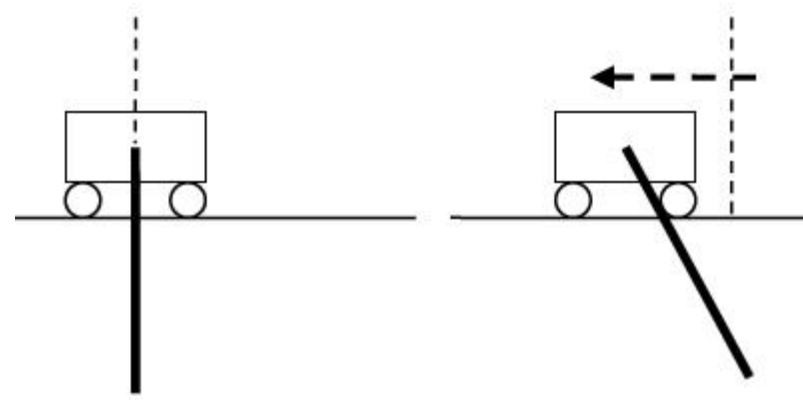

Source: elaborated by the authors.

Figure 6 - Second cart motion

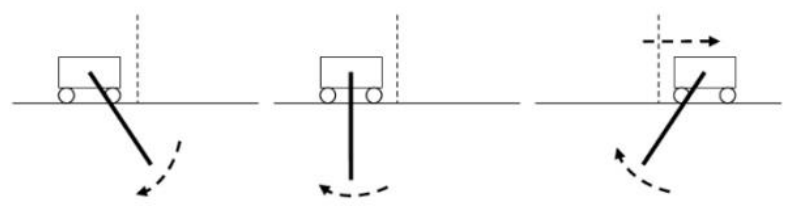

Source: elaborated by the authors.

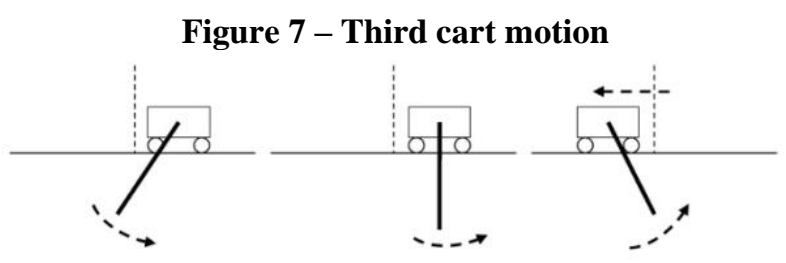

Source: elaborated by the authors.

The procedure above described is repeated until the angle between the pendulum and vertical is equal to or less than five degrees. At this moment the system switches for inverted pendulum position control, which assumes the command of cart motion. This knowledge will be translated into a set of rules, and for each variable of interest, a universe of discourse with fuzzy membership functions will also be developed.

Based on the above heuristics, the following membership functions for the input variables were established:

- Angle $\theta$ Figure 8:
Figure 8 - Angle $\theta$ membership functions

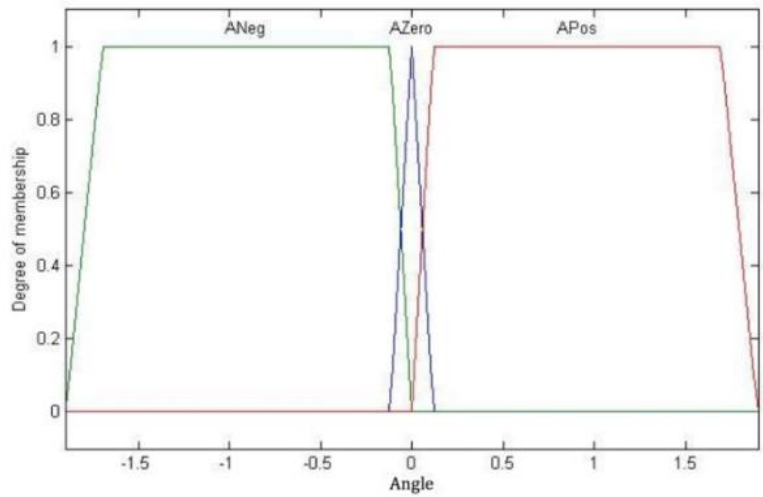

Source: elaborated by the authors.

For the input $\theta$ there are three membership functions as shown in Figure 8: ANeg, trapezoidal for negative angles; AZero, triangular for angles smaller than five degrees; and APos, trapezoidal for positive angles.

- Angular speed $\dot{\theta}$ Figure 9

Figure 9 - Angular speed $\dot{\theta}$ membership functions

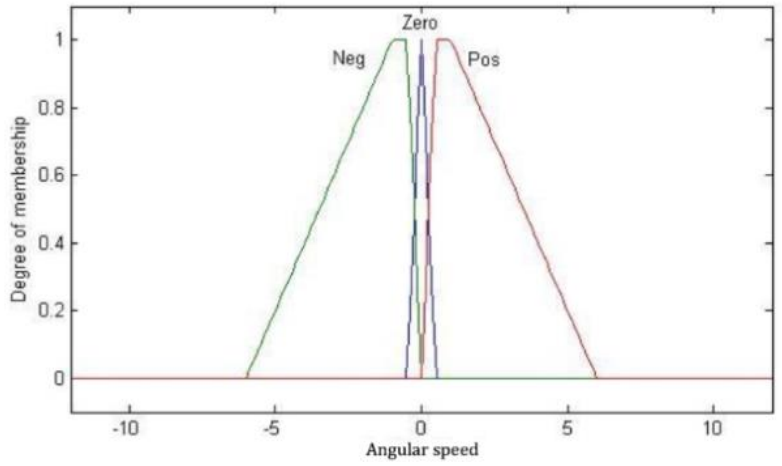

Source: elaborated by the authors.

For the input $\dot{\theta}$ there are also three speed membership functions as shown in Figure 9: ANeg, trapezoidal for negative; AZero, triangular for small; and APos, trapezoidal for positive speeds.

The membership functions of the output $x_{C}$ used at the defuzzification phase are shown in Figure 10. 
Figure 10 - Position $x_{C}$ membership functions

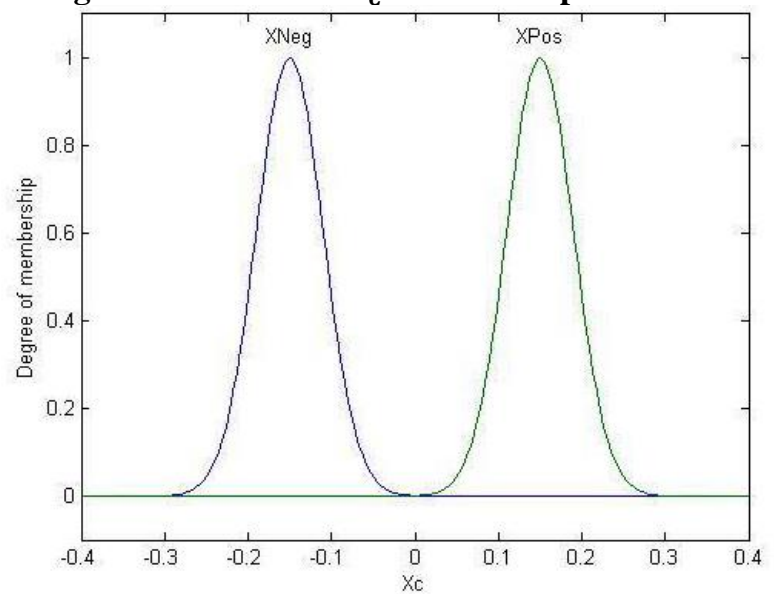

Source: elaborated by the authors.

Both membership functions are gaussian, but they could be triangular, being their peak values the most important feature.

These variables and their respective membership functions are shown in Table 1.
Table 1 - Fuzzy Controller Rules

\begin{tabular}{llllll}
\hline If & \multicolumn{1}{c}{$\theta$} & And & \multicolumn{1}{c}{$\dot{\theta}$} & Then & \multicolumn{1}{c}{$x_{C}$} \\
\hline If & Zero & And & Zero & Then & Neg. \\
If & Zero & And & Negative & Then & Posi. \\
If & Zero & And & Positive & Then & Neg. \\
If & Negative & And & Negative & Then & Posi. \\
If & Positive & And & Negative & Then & Neg. \\
If & Negative & And & Positive & Then & Posi. \\
If & Positive & And & Positive & Then & Neg. \\
If & Negative & And & Zero & Then & Posi. \\
If & Positive & And & Zero & Then & Neg. \\
\hline
\end{tabular}

Source: elaborated by the authors.

\section{SIMULATION ENVIRONMENT}

Figure 11 shows the Simulink ${ }^{\circledR}$ block diagram for the swing up process. The swing up control is implemented in four steps as shown: angle pendulum conversion in orange; fuzzy inference engine in blue; sigmoid function in green; and position control in red.

Figure 11 - Pendulum Swing Up Fuzzy Control

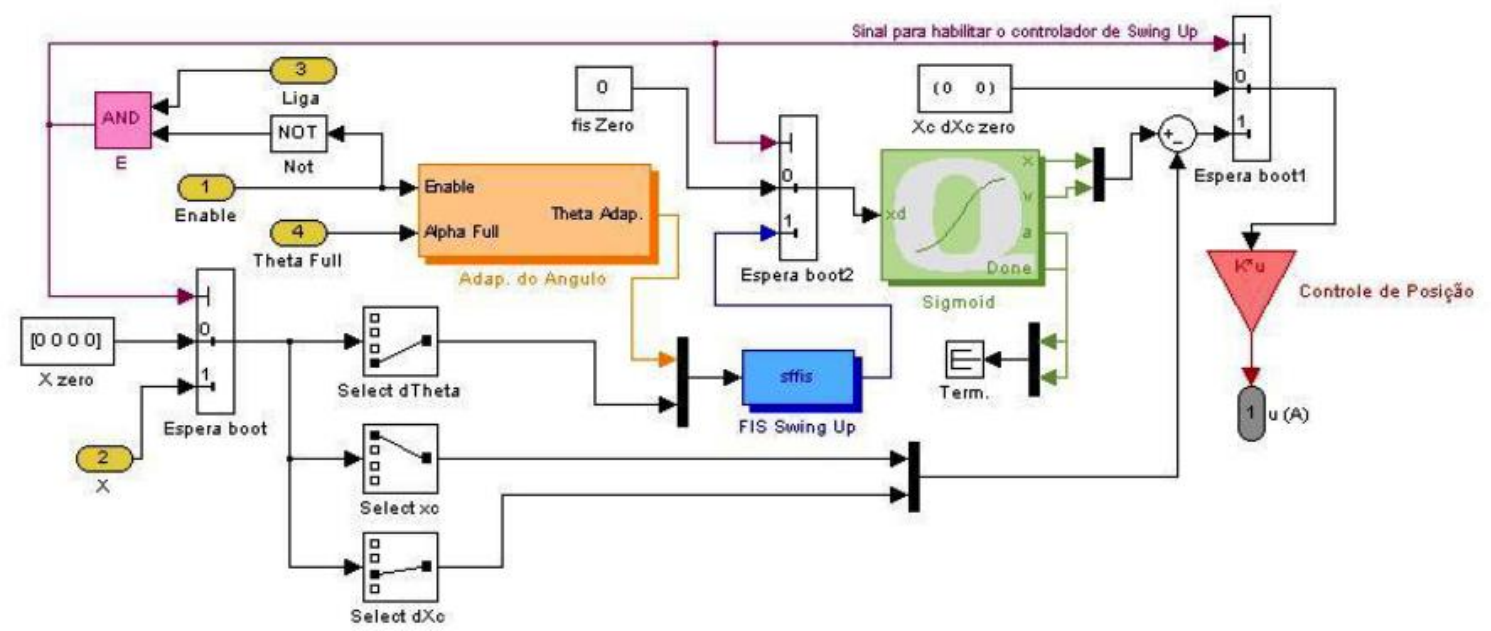

Source: elaborated by the authors.

The orange block in Figure 11, pendulum angle conversion, modifies the pendulum angle $\theta$ so that the fuzzy inference engine can correctly interpret it. The angle conversion block was built so that it limits $\theta$ universe of discourse to the interval $[-\pi, \pi]$, otherwise, it should be $[-\infty,+\infty]$. The structure of the pendulum angle conversion block can be seen in Figure 12.

Besides defining the angle $\theta$ universe of discourse, one can see in Figure 12 that this is not the only function of this block. It also makes $\theta$ be always positive in the first and second quadrants at the interval $[0, \pi]$, no matter how many turns it may have done until it reaches these quadrants. Figure 13 shows the pendulum at a positive quadrant. 
Figure 12 -Pendulum Angle Conversion Block

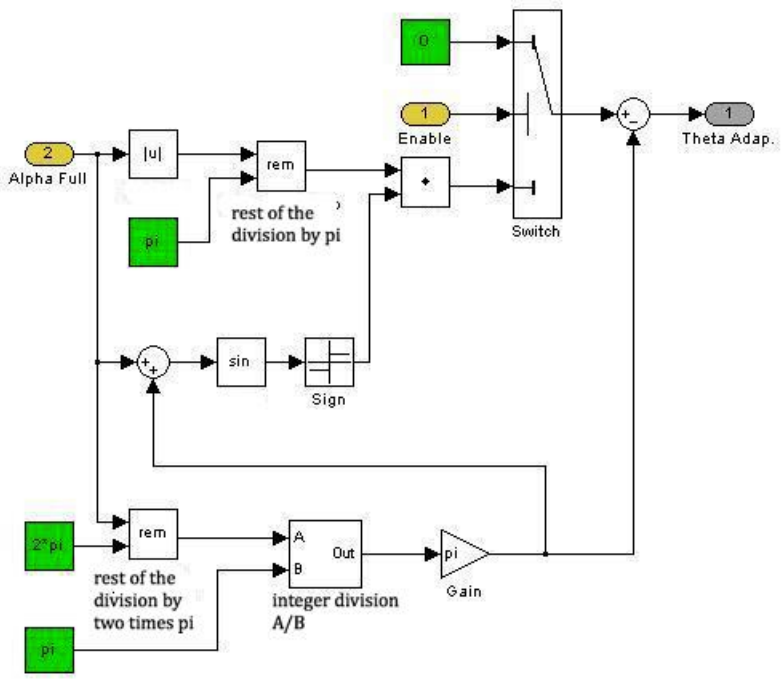

Source: elaborated by the authors.

Figure 13 - Positive 1st and 2nd Quadrants

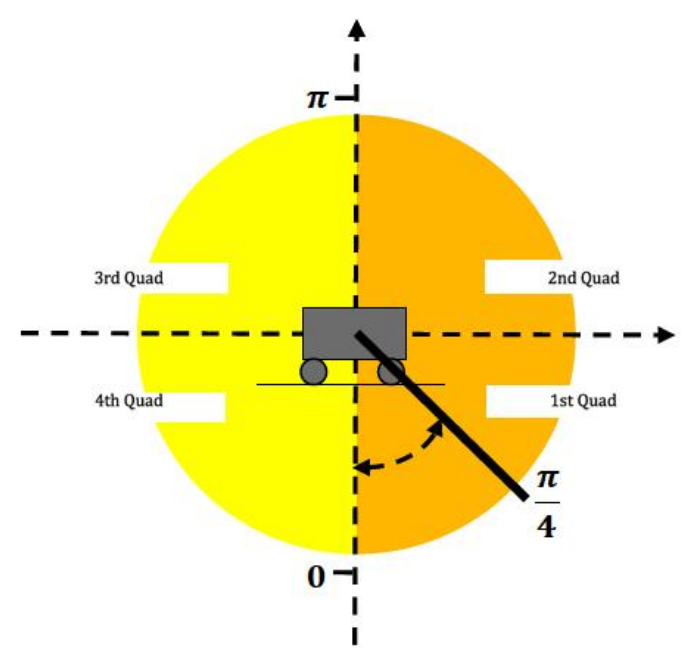

Source: elaborated by the authors.

When the pendulum is in the third or fourth quadrant, the algorithm brings its angle to the interval $[-\pi, 0]$, no matter how many turns it may have done. This can be shown in Figure 14.

In this way, the Pendulum Angle Conversion Block ensures that no matter how many turns the pendulum may have done, its angle will be always in the interval $[-\pi, \pi]$, thereby making it possible to establish the fuzzy rules.
Figure 14 - Negative 3rd and 4th Quadrants

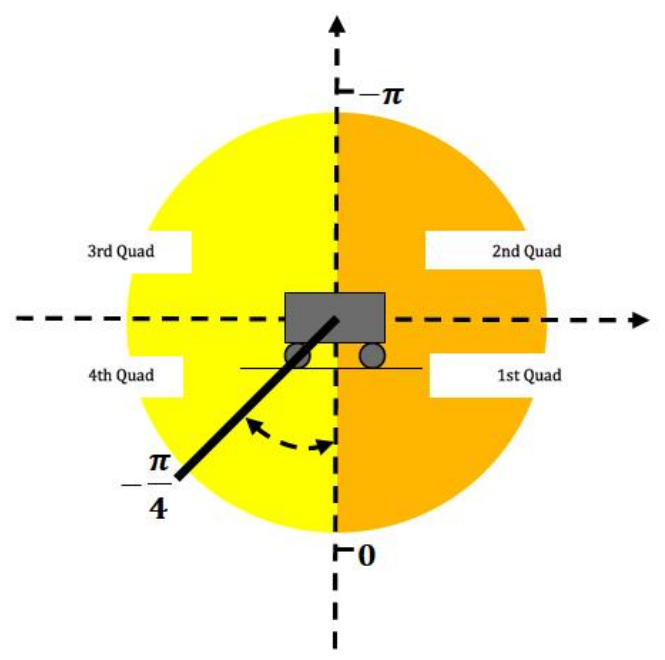

Source: elaborated by the authors.

As shown in Figure 11, the FIS Swing-Up blue block is a standard MatLab ${ }^{\circledR}$ called SFunction, which constitutes the Fuzzy Inference System. It is developed for the: input variable fuzzification; rule application; and defuzzification of the rule output.

The red block in Figure 11 is the cart position reference command for a proportionalderivative (PD) control implemented by the manufacturer (QUANSER, 2008). The inputs are the position and speed errors, and the output $u$ is the current applied to the HFLC cart motor.

The only output of the fuzzy controller is the reference command for the cart position control. The Sigmoid function act as a smoother for the fuzzy output, therefore avoiding current peaks at the cart electric motor and ensuring a correct command following.

After the swing-up phase, the system is switched for an LQR controller that keeps the pendulum in the inverted position, as soon as it is within a limit of plus or minus five degrees from the vertical.

\section{SIMULATION RESULTS}

In order to verify the performance of the fuzzy control, the non-linear model of the cartpendulum system according to Equation (23) is implemented in Simulink®. 
Figure 15 shows the cart displacement $x_{C}$ and the pendulum angle $\theta$ with respect to time. The fuzzy logic swing-up system controls the cart motion in the time interval [0 5.112s] until the pendulum reaches its inverted position. From the instant $5.112 s$, the control system changes to the pendulum stability control mode, which keeps it always upright inverted.

Figure 15 - Time plot of $x_{c}$ and $\theta$
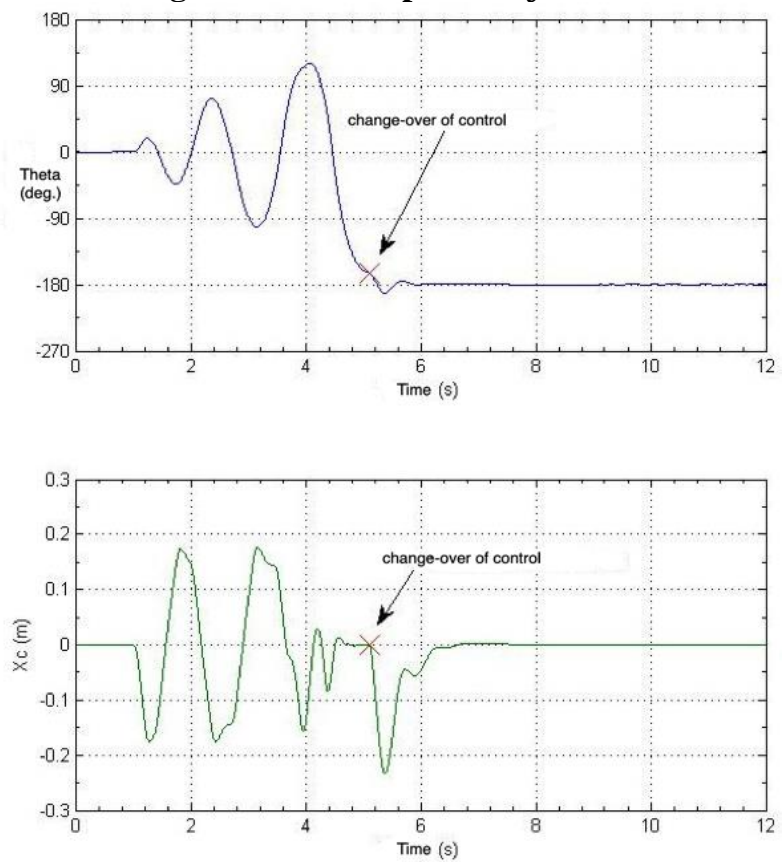

Source: elaborated by the authors.

After the system simulation in the SimuLink ${ }^{\circledR}$ is completed, the next step consists of the Hardware-in-the-loop simulation (HILS) using the complete hardware of the Quanser HFLC (QUANSER, 2008).

Figure 16 shows the cart motion and the pendulum angle during the experiment of simulation with the hardware in the loop. During the time interval $[0$ 5.977)s the swingup fuzzy controller caused the pendulum to turn 360 degrees before moving to the inverted position, after which it was stabilized in this state.

The system did not behave as expected due to the following reasons:

- the tuning of the fuzzy controller was based on a non-linear mathematical model, equation (23), that didn't include the actuator dynamics of the force $F$ acting on the cart;
- there isn't a base of rules to control the potential energy and angular speed $\dot{\theta}$ of the pendulum, therefore avoiding the overshoot at the top position, nevertheless the system was able to perform the swing-up phase.

Figure 16 - HILS - Time plot of $x_{C}$ and $\theta$
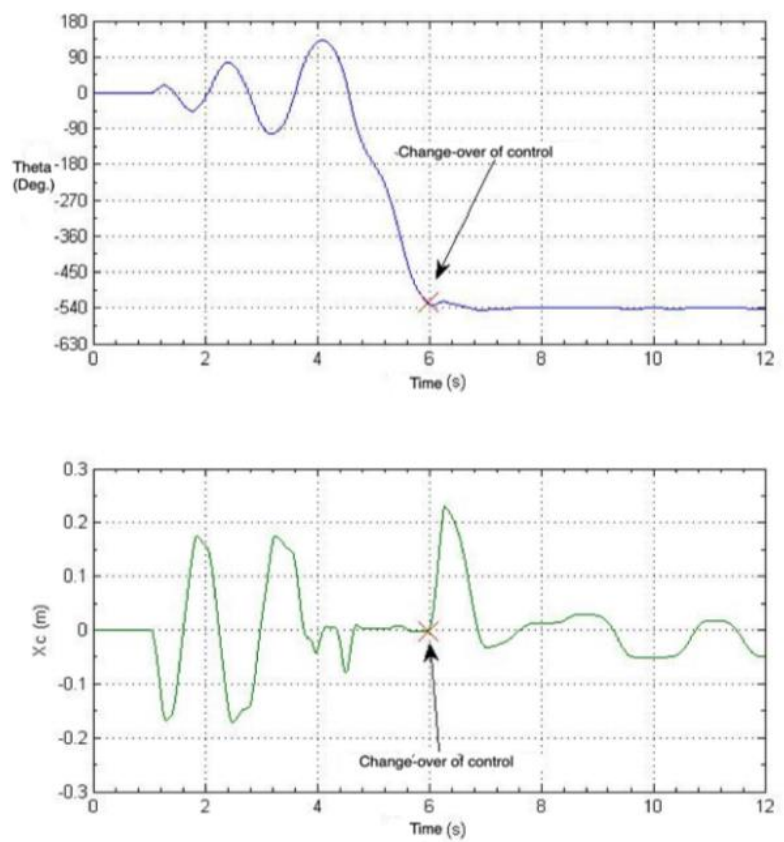

Source: elaborated by the authors.

\section{CONCLUSION}

\section{Educational goals achieved}

The use of an Inverted Pendulum System hardware enabled the following results to be attained by the team of students:

- Inverted pendulum modelling.

The team was able to deal with a complex electro-mechanical system applying the Lagrangian Theory (Eq. 1) to obtain the statespace non-linear model (Eq. 23) that approximately describes the behavior of the system.

- Actuator dynamics.

The force $F$ acting on the HFLC System cart (QUANSER, 2008) is provided by a step motor connected to a gear train, which causes the trolley to move along a rack. Therefore, the step 
motor dynamics, gear backlashes nonlinearities, and time delays were not taken into account by the team in the system modeling, contributing to the unexpected behavior in the closed-loop configuration.

- Energy management.

The team was able to recognize the need for a set of rules that deal with the potential energy \$V\$ of the system, according to equation (2), reducing the motion amplitude as the pendulum approaches the top position.

- Intelligent control system.

The team was able to learn and implement the fundamentals of Intelligent Control by applying the principles of the Fuzzy Logic Theory to complex hardware. The fuzzy rule base was developed by observation of a human operator performing the swing-up task by hand.

- Fuzzy logic controller model based tuning.

The swing-up fuzzy controller was tuned using a non-linear mathematical model in SimuLink®, what clearly shows how mathematical modeling limitations and errors affect the performance, addressing the need for robust control techniques to deal with modeling uncertainties, including an unmodelled actuator, and high-frequency dynamics.

- Fuzzy Logic Control Robustness.

Even though the mathematical modeling was not able to capture the actuator dynamics and the parametric uncertainty of the system, the students realized the robustness characteristics of the fuzzy logic controller, which was able to perform the swing-up motion.

\section{Future developments}

The teaching of Intelligent Control Systems subject will take full advantage of the inverted pendulum hardware by establishing the following educational goals for the students:

- Improving the non-linear model by including the actuator dynamics, timedelays, pinion and rack backlashes, and other nonlinearities;
- Implementing additional rules to reduce the motion amplitude as the pendulum approaches the top position;

- Substituting the Linear Quadratic Regulator that keeps the pendulum in its inverted position, by a set of fuzzy rules, so that students become able to implement a full fuzzy logic controller.

\section{REFERÊNCIAS}

BLOCK, D. J.; ÄSTRÖM, K. J.; SPONG, M. W. The Reaction Wheel Pendulum. [S.1.]: Morgan \& Claypool Publishers, 2007.

LIMA, G. B. D. A.; BELARMINDO, J. N. Modelagem de controladores para sistemas não lineares através de inteligência artificial: $O$ pêndulo invertido. CEFET-RJ. Nova Iguaçu, 2011.

MERIAM, J. L. Dynamics. New York: John Wiley \& Sons, Inc., 1966.

QUANSER. High Fidelity Linear Cart (HFLC), User Manual. Ontario: Quanser, 2008. 


\section{AUTHORS' BIOGRAPHICAL DATA}
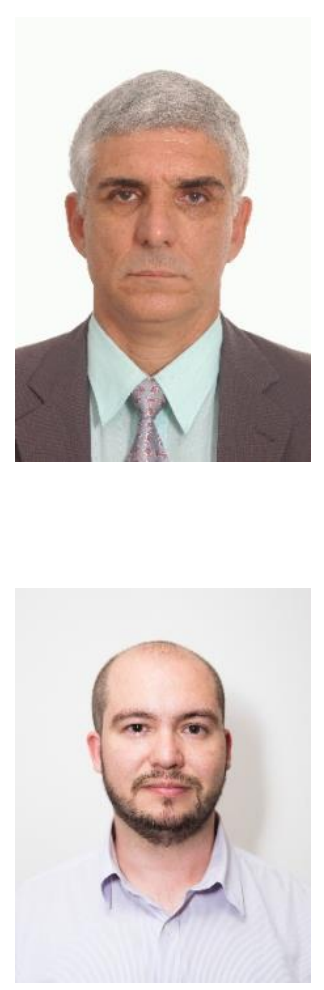

Alexandre Alves Santiago - Doutor em Engenharia Oceânica pela Universidade Federal do Rio de Janeiro (2008). Master of Science in Aeronautics and Astronautics pelo Massachusetts Institute of Technology (1992), Engenheiro Naval pela Universidade de São Paulo (1984). Atualmente é professor de Engenharia Mecânica nas áreas de Ensino Básico, Técnico e Tecnológico, no Centro Federal de Educação Tecnológica Celso Suckow da Fonseca (CEFET-RJ).

Glauber Bolzan de Assis Lima -Engenheiro de Controle e Automação pelo Centro Federal de Educação Tecnológica Celso Suckow da Fonseca (CEFET-RJ). 